\title{
Claves de los conflictos socioambientales mexicanos
}

\section{Darcy Tetreault}

Resumen. Políticos e investigadores asumen que el crecimiento económico es imprescindible para reducir la pobreza, puesto que redunda en la creación de empleos y de financiamiento de las políticas sociales. Si bien en la posguerra el crecimiento contribuyó a reducir la pobreza, las políticas sociales ya no pueden subordinarse al imperativo del crecimiento económico debido a los límites ecológicos y los conflictos socioambientales. De ahí la relevancia de pensar en alternativas poscapitalistas. En las últimas décadas se advierte que la dinámica de acumulación de capital ha generado rupturas metabólicas en el nivel planetario y múltiples problemas ambientales en los ámbitos nacional y local. Las propuestas de decrecimiento postulan el posdesarrollo a escalas local y subnacional y el posestructuralismo enfocado en el discurso, los valores y la cultura. La perspectiva marxista sobre el decrecimiento alude a los cambios estructurales necesarios y la cuestión de agencia. Los conflictos socioambientales en México se han multiplicado desde la aplicación en 1992 de reformas estructurales diseñadas para liberar la tierra, el agua y los demás recursos naturales a las fuerzas del mercado. Las demandas, propuestas y alternativas de las luchas socioambientales mexicanas constituyen puntos de referencia insoslayables para pensar en las formas de organización y las políticas públicas requeridas con el fin de empezar una transición hacia modos de producción y gobernanza que exhiben racionalidad socioecológica y diversidad biocultural, y que están orientados a la satisfacción de las necesidades básicas de todo el mundo.

Palabras clave: conflictos socioambientales, límites ecológicos, poscapitalismo, posdesarrollo, México.

* Docente investigador, Unidad Académica en Estudios del Desarrollo, Universidad Autónoma de Zacatecas, México. 


\section{Key features of Mexican socio-environmental conflicts}

Abstract. Politicians and researchers assume that economic growth is essential in order to reduce poverty, as it is based on the creation of jobs and the funding of social policies. Although in the post-war era growth contributed to poverty reduction, social policies can no longer be subordinated to the imperative of economic growth, given that there are ecological limits and socio-environmental conflicts. Thus appears the relevance of thinking about post-capitalist alternatives. In the past few decades, concerns have developed regarding the dynamics of capital accumulation that have contributed to metabolic ruptures at the global level and with multiple environmental problems in national and local spheres. The proposals for de-growth conceive of a post-development process at local and sub-national levels, and a post-structuralism focused on discourse, values and culture. The marxist perspective on de-growth alludes to the necessary structural changes and the question of agency. Socio-environmental conflicts in Mexico have multiplied since the 1992 application of structural reforms designed to put land, water and other natural resources at the mercy of market forces. The demands, proposals and alternatives of Mexican socio-environmental struggles constitute key points of reference for the conception of forms of organization and public policies required to transition toward modes of production and governance that demonstrated socio-ecological rationality and bio-cultural diversity, and which are directed toward the satisfaction of basic needs for all.

Keywords: socio-environmental conflicts, ecological limits, post-capitalism, postdevelopment, Mexico. 
Durante el tercer debate presidencial que se llevó a cabo el 12 de junio de 2018, el candidato del partido Movimiento Regeneración Nacional (Morena), Andrés Manuel López Obrador, señaló que la falta de crecimiento económico estaba en el trasfondo de los grandes problemas sociales del país. En sus palabras: «Si no hay crecimiento, no hay empleos. Si no hay empleos, no hay bienestar. Si no hay bienestar, no hay paz, no hay tranquilidad». López Obrador, como muchos investigadores que se especializan en los temas de pobreza y desigualdad y en el análisis de las políticas sociales, da por sentado que el crecimiento económico es una condición sine qua non para reducir la incidencia de pobreza, ya que se traduce en la creación de empleos y en la generación de financiamiento para la expansión de políticas sociales.

Se argumenta en este texto que, si bien esta condición contribuyó a reducir la incidencia de pobreza durante el periodo de la posguerra, las políticas sociales ya no pueden ser subordinadas al imperativo de lograr crecimiento económico, debido a los límites ecológicos y conflictos socioambientales que se han manifestado en distintos niveles. Tomando en cuenta que una economía capitalista que no crece cae en crisis con impactos negativos, sobre todo para los trabajadores y los sectores marginados de la población, se plantea la necesidad de pensar en alternativas poscapitalistas.

La primera sección examina los debates en torno a los límites del crecimiento, se llega a la conclusión de que la dinámica de acumulación de capital durante las últimas décadas ha resultado en una serie de rupturas metabólicas a nivel planetario y múltiples problemas ambientales en los ámbitos nacional y local. La segunda sección analiza diversas propuestas de decrecimiento y distingue entre dos acercamientos interrelacionados: uno posdesarrollista, inspirado por el pensamiento de Iván Illich y 
enfocado en las escalas local y subnacional; y otro posestructuralista, enfocado en el discurso, los valores y la cultura. La tercera sección entra en diálogo con las aportaciones marxistas a los debates en torno al decrecimiento y retoma algunas de sus propuestas con respecto a los cambios estructurales necesarios y la cuestión de agencia.

La cuarta sección analiza los conflictos socioambientales en México que se han multiplicado desde la aplicación en 1992 de reformas estructurales diseñadas para liberar la tierra, el agua y los demás recursos naturales a las fuerzas del mercado. En la misma sección, se analizan algunas de las demandas y propuestas de las redes que operan a nivel nacional y que reúnen distintas luchas socioambientales a lo largo del país, así como las experiencias en los ámbitos local y regional que tienen cierta resonancia con el posdesarrollo, sobre todo en comunidades indígenas. El texto termina con una serie de reflexiones y conclusiones, donde se plantea inter alia que las demandas, propuestas y alternativas de las luchas socioambientales mexicanas constituyen puntos de referencia insoslayables para pensar en las formas de organización y las políticas públicas requeridas para empezar una transición hacia modos de producción y gobernanza que exhiben racionalidad socioecológica y diversidad biocultural, y que están orientados a la satisfacción de las necesidades básicas de todo el mundo.

\section{Los límites al crecimiento económico}

¿Existen límites ecológicos absolutos al crecimiento económico? Sobre este tema hay debate. Según los optimistas tecnológicos se puede realizar crecimiento económico indefinidamente, superando los límites de la 
naturaleza a través de la innovación tecnológica, la sustitución entre diferentes materiales y el descubrimiento de nuevas reservas. En esta visión, los mercados dan las señales e incentivos necesarios para impulsar los procesos de innovación, sustitución y descubrimiento; el Estado nación y el sector privado son los actores centrales en un esfuerzo coordinado a nivel internacional por mejorar el manejo de los recursos naturales y hacer más ecológicamente eficientes los procesos de producción, distribución y consumo, con el objetivo de extender cada vez más la capacidad de los ecosistemas de proveer materias primas y absorber los desechos.

Ésta es esencialmente la visión de «desarrollo sostenible» esbozada en el Informe de Brundtland (WCED, 1987) e institucionalizada a nivel mundial por la Organización de Naciones Unidas (ONU) y otros organismos internacionales de desarrollo a partir de la primera Cumbre de la Tierra en Río de Janeiro en 1992. El Informe de Brundtland no reconoce límites absolutos al crecimiento, sólo «límites impuestos por el estado de la tecnología y la organización social sobre la capacidad del medio ambiente para satisfacer las necesidades presentes y futuras» (WCED, 1987:43). El mismo Informe señala la necesidad de realizar tasas de crecimiento de por lo menos 3 por ciento anual en todas partes del mundo (WCED, 1987:50-51). Asimismo, el preámbulo de la Agenda 21 pone énfasis en la necesidad de contar con un crecimiento económico internacional dinámico y confiable y subraya los méritos del libre comercio al respecto. Más recientemente, la Agenda 2030 para el Desarrollo Sostenible de la ONU no duda en la necesidad de realizar crecimiento económico, mientras aboga por hacerlo más inclusivo y sostenible. Según el Objetivo 12 de esta Agenda, el consumo y la producción sostenible «consisten en fomentar el uso eficiente de los recursos y la energía». 
Los escépticos frente a la posibilidad de realizar crecimiento económico indefinido expresan múltiples argumentos desde diversas posturas políticas y epistemológicas. Para empezar, desde finales de los años sesenta del siglo pasado una corriente de pensamiento neomaltusiano ha encontrado expresión en publicaciones populares, tales como The population bomb (Ehrlich, 1968) y The limits to growth (Meadows et al., 1972). Para esta corriente, el meollo del argumento es simple: en un mundo finito ninguna cosa material puede crecer exponencialmente, incluso el número de seres humanos y el consumo de recursos naturales no renovables.

Al mismo tiempo, emergió una versión más sofisticada del argumento de que existen límites absolutos al crecimiento: Georgescu-Roegen (1972) recurrió a la segunda ley de la termodinámica para argumentar que la extracción y el consumo de minerales y combustibles fósiles no pueden continuar de manera indefinida. Mientras históricamente el desarrollo industrial ha dependido cada vez más de ellos, implica el agotamiento de materiales con bajos niveles de entropía y su transformación irreversible a formas de contaminación con altos niveles de entropía. Cabe señalar que el trabajo de Georgescu-Roegen sigue siendo un punto de referencia importante para los defensores del decrecimiento.

Durante los años noventa, cuando todavía era posible ser optimista sobre la Agenda 21 y la noción dominante de desarrollo sostenible, emergió en el campo de la economía ambiental la idea de que se puede observar una especie de curva invertida de Kuznets en la relación entre los niveles de desarrollo y el deterioro del medio ambiente. En este modelo, que buscaba su soporte empírico en un análisis sincrónico de múltiples países categorizados por su nivel de desarrollo y basado en la suposición de que todos siguen un camino unidireccional de progreso definido por 
la experiencia histórica de los países del Norte global (véase por ejemplo, Panayotou, 1995), el medio ambiente se deteriora durante las primeras fases de desarrollo, para posteriormente mejorar después de cierto punto de inflexión, cuando las economías «se modernizan». Esto, según los defensores de la curva, se debe a múltiples factores, entre los más importantes: la creciente importancia relativa de los sectores de servicios e información en las economías modernas, los cuales consumen menos materiales y energía; un cambio de valores conforme se satisfacen las necesidades básicas de grandes sectores de la población; y la disponibilidad de más recursos para financiar políticas ambientales.

No obstante, como han señalado los escépticos al respecto (por ejemplo, Stern, 1998; Ekins, 2000; Dinda, 2004), la evidencia empírica sugiere que esta relación sólo es válida por un número limitado de indicadores, generalmente los asociados con algunas formas de contaminación del aire, no puede ser generalizada a todos los aspectos de la calidad ambiental; además, varias formas de contaminación y los gases de efecto invernadero aumentan con la industrialización y el crecimiento del producto interno bruto (PIB) (Martínez Alier, 1994:87-88). Los avances tecnológicos y los sustitutos «establecen la posibilidad teórica, no la certeza, ni la probabilidad y ni siquiera la posibilidad en la práctica de que el crecimiento continúa indefinidamente» (Lecomber citado en Ekins, 2000:4). En efecto, como señaló Sachs (1993:16) desde hace más de un cuarto de siglo, los sectores de servicios e información «sólo pueden crecer por encima del sector industrial y en asociación estrechamente simbiótica».

Para Martínez Alier (2011:38), el desarrollo sostenible apuesta por el «evangelio de la ecoeficiencia», o sea «una religión de la utilidad y la eficiencia técnica sin una noción de lo sagrado». Esta es la apuesta detrás de 
el Objetivo 12 de la Agenda 2030 de la ONU: hacer más ecológicamente eficiente el uso y consumo de recursos naturales. Como han señalado múltiples autores, uno de los principales problemas con esta corriente de pensamiento es que ignora la paradoja de Jevons, la cual postula que los incrementos en la eficiencia con que se utiliza o consume un recurso conducen a crecientes niveles de demanda y consumo, lo que eclipsa, efectivamente, los avances en mejorar la eficiencia (véase, por ejemplo, Foster, 2002:94-95; Latouch; 2008:46; Foster et al., 2010:169-181; Schneider et al., 2010:516; Wi1liams, 2010:198-199; Martínez Alier, 2011:28; Kallis, 2017).

En efecto, aunque no existe una ley intrínseca basada en una teoría económica que demuestra lógicamente que el crecimiento económico y el flujo de energía y materiales siempre estarán vinculados en una economía capitalista (Kallis, 2017), la evidencia empírica revela esta tendencia. Por ejemplo, en un estudio sobre el uso de materiales a nivel global, Krausmann et al. (2009:2701) observan que durante el siglo XX el «metabolismo social» — es decir, la tasa de flujo de materiales y energía — creció dos veces más rápido que la población y ha acelerado a partir de finales de los años noventa. Así, a pesar de los impresionantes incrementos en la eficiencia con la que se producen y consumen los materiales y la energía, el metabolismo social global sigue siendo ascendente. Más aún, se puede observar un flujo neto de materiales y energía desde el Sur hacia el Norte (con las excepciones notables de Australia y Canadá, dos países desarrollados que son exportadores netos) (Dittrich y Bringezu, 2010), lo que evidencia la persistencia de un «comercio ecológicamente desigual» (Delgado Ramos, 2012; Martínez Alier y Walter, 2016).

Desde una perspectiva marxista, «el problema no es la sobrepoblación: es que bajo las relaciones sociales capitalistas se empuja a la gente a 
expandir rápidamente la producción orientada al mercado para realizar ganancias» (Williams, 2010:43). Marx, en su polémica contra Malthus, argumentó que «la sobrepoblación es (...) una relación determinada históricamente, de ninguna manera determinada por los números abstractos o por el límite absoluto de la productividad de lo necesario para la vida, sino más bien por límites establecidos por condiciones específicas de producción» (énfasis en el original, Marx, 1973:606). Si bien esta postura sobre la no existencia de límites absolutos al crecimiento puede parecer a primera vista la misma expresada por el Informe de Brundtland, la diferencia es que para Marx dichas condiciones se refieren no sólo a aspectos tecnológicos y técnico-organizacionales sino también y más importante al modo dominante de producción. Marx observó que «la producción capitalista sólo sabe desarrollar la técnica y la combinación del proceso social de producción socavando al mismo tiempo las dos fuentes originales de toda riqueza: la tierra y el hombre» (Marx, 1977:638).

Marx era consciente de los impactos destructivos del desarrollo capitalista sobre el medio ambiente. Su visión histórico-materialista contempla una interacción dialéctica y mutuamente transformadora entre la sociedad humana y el medio ambiente, mediada por el trabajo. De esta manera, teoriza una «ruptura metabólica» a partir de la subsunción del trabajo y la naturaleza a los procesos productivos orientados a crear valor de cambio. Con base en el trabajo del agroquímico Justus von Liebig, Marx analizó las consecuencias de esta «ruptura metabólica» en la pérdida de fertilidad de la tierra, entre otros problemas ambientales.

John Bellamy Foster (2000), además de revindicar «la ecología de Marx» (como reza el título de su libro), ha recuperado el concepto de la ruptura metabólica y lo ha desarrollado para poner de relieve su relevancia 
en la actualidad. Sobre esta línea, postula que durante la segunda mitad del siglo XX sucedió una profundización de la ruptura metabólica en el sector agrícola en la medida en que se concentró la producción de ganadería cerca de las plantas de procesamiento, separándola así aún más de la producción agrícola especializada en cultivos (Foster, 2002:162-166). De esta manera, el estiércol de los animales, en lugar de servir como fertilizante, se convierte en una fuente de contaminación y conflictividad socioambiental, como en el caso de Granjas Carroll de México (propiedad de Smithfield Foods) en el Valle del Cofre de Perote, en el estado de Veracruz.

En un libro más reciente con Brett Clark y Richard York (2010:13-19), Foster y sus colaboradores señalan nueve límites planetarios o «rupturas» que han sido analizados por un grupo de investigadores en el Stockholm Resilience Center: el cambio climático, la pérdida de biodiversidad, los ciclos de nitrógeno y fósforo, la acidificación oceánica, el agotamiento del ozono estratosférico, el agotamiento y la contaminación del agua dulce, el cambio de uso de suelo, el deterioro atmosférico relacionado al uso de aerosoles, y la contaminación química. Según este grupo de científicos, los primeros tres límites están en su punto de quiebre y los otros seis significan el inicio de degradación ambiental irreversible. Desde una perspectiva marxista, esta evidencia empírica apoya la hipótesis de que el capitalismo de por sí no es sustentable.

James O’Connor (1998) llega a esta conclusión a través de su análisis de las dos contradicciones básicas en el desarrollo capitalista que empujan el sistema hacia las crisis. La primera, expuesta por Marx, se encuentra en la relación entre el capital y la fuerza de trabajo, «cuando capitales individuales tratan de defender o restaurar las ganancias al incrementar la productividad laboral, acelerando el trabajo, reduciendo los salarios y 
utilizando otras maneras de larga data para sacar más producción de menos trabajadores» (1998:240). El resultado no intencional es de reducir la demanda por los bienes consumibles, lo que inclina el sistema hacia una crisis de sobreproducción.

O'Connor (1998) propone que existe una segunda contradicción. Ésta afecta el lado de los costos, cuando el capital socava las «condiciones de producción», las cuales incluyen condiciones naturales, comunales y humano-laborales. Hay dos maneras de hacerlo: «Cuando los capitales individuales defienden o restauran las ganancias a través de estrategias que degradan o no logran mantener con el tiempo las condiciones materiales de su propia producción» $\mathrm{y}$ «cuando los movimientos sociales demandan que el capital provea mejor para el mantenimiento y restauración de estas condiciones de vida» (1998:242). La posibilidad de que el capital internalice los costos ambientales y reconstruya las condiciones de producción mediante la regulación estatal para restaurar o aumentar su productividad a largo plazo es remota, argumenta O'Connor, puesto que se requeriría la coordinación de grandes inversiones y la renuncia de ganancias a corto plazo, algo que va en contra de la lógica autoexpansiva y antiecológica del capital (1998:246).

\section{Estado estacionario y decrecimiento}

Desde los años setenta, Herman Daly ha abogado por una transición hacia una economía estacionaria, definida por cantidades constantes de riqueza física, una población humana no creciente, y una tasa baja del flujo de energía y materiales. Su visión se inspira en el estado estacionario imaginado por John Stuart Mill a mediados del siglo XIX. A diferencia 
de la economía neoclásica, Daly (1996) insiste en que las actividades económicas humanas constituyen un subsistema del ecosistema planetario donde la segunda ley de la termodinámica establece límites biofísicos al crecimiento del uso y consumo de materiales y energía. Además, hace una distinción entre crecimiento y desarrollo. El primero «se refiere a un aumento en la escala física del flujo de materiales y energía que sostiene las actividades económicas de la producción y consumo de bienes» (Daly, 1996:31). El segundo puede ser concebido en términos cualitativos como una mejora en la calidad de vida que no necesariamente implica un aumento en el flujo de materiales. Así, mientras existen límites al crecimiento económico, no hay límites al desarrollo. La solución para Daly (1996:7) «es dolorosamente sencilla: controlar la población, redistribuir la riqueza y los ingresos, y realizar mejoras técnicas en la productividad de los recursos». Sugiere que el Banco Mundial y los Estados nación son las agencias clave en realizar esta transición y apela a argumentos religiosos y principios éticos para guiar la reformulación de la política pública.

Los defensores del decrecimiento van más lejos al abogar a favor del achicamiento de la economía en el Norte global. En las palabras de Serge Latouche (2008:15), el representante más reconocido a nivel mundial de esta escuela de pensamiento, «no se trata ni del estado estacionario de los viejos clásicos, ni de una forma de regresión, de recesión ni de «crecimiento negativos, ni incluso de crecimiento cero - aunque encontramos en él una parte de la problemática». El decrecimiento, entonces, tiene que ser distinguido en primer lugar de las recesiones y crisis económicas, cuyas consecuencias son devastadoras para los trabajadores: «Distinguimos entre depresión, es decir decrecimiento no planeado dentro de un régimen de crecimiento, y decrecimiento sustentable, una transición voluntaria, lisa y equitativa a un 
régimen de menos producción y consumo» (Schneider et al., 2010:511). En última instancia, esta visión de decrecimiento es compatible con la del estado estacionario, ya que una economía no puede decrecer indefinidamente, de tal manera que tarde o temprano tendrá que establecer un estado estacionario para ser sustentable (Martínez Alier et al., 2010:1744).

Ambas corrientes de pensamiento (decrecimiento y estado estacionario) toman en serio la paradoja de Easterlin, la cual postula que el crecimiento económico a nivel nacional no conlleva después de cierto punto a un aumento en la felicidad de los ciudadanos. Asimismo, ambas corrientes critican la contabilidad del PIB, entre otras razones porque no toma en cuenta el trabajo no remunerado para la reproducción social, como han señalado las feministas desde hace décadas; porque no descuenta la disminución del «capital natural» (es decir, minerales, hidrocarburos, fertilidad del suelo, bosques, acuíferos, etcétera) y porque registra como algo positivo los gastos defensivos, incluso los asociados con la protección y rehabilitación de los ecosistemas. Daly (1996:99-102) aboga por una contabilidad que pretende corregir estas deficiencias: el producto nacional neto sustentable. Verma (2017:487) argumenta a favor de la Felicidad Nacional Bruta, ${ }^{1}$ la medición adoptada por el gobierno del Reino del Himalaya de Bután, que «no sólo es congruente con el decrecimiento, sino también un ejemplo vivo del mismo». Mientras Latouche (2008) pregunta si no sería más sensato «salir de la obsesión de medirlo todo». Desde su punto de vista, «el problema no es cambiar el patrón de medida para transformar

${ }^{1}$ La Felicidad Nacional Bruta se mide a través de un índice compuesto de indicadores en nueve áreas: salud, educación, estándares de vida, uso de tiempo, gobernanza, diversidad y resistencia ecológica, bienestar psicológico, vitalidad comunitaria, y diversidad y resistencia cultural (Verma, 2017:482). 
la sociedad, sino empezar a cambiar los valores» a través de «la descolonización del imaginario» (Latouche, 2008:82). He aquí uno de los supuestos cuestionables de la visión de decrecimiento de Latouche, a saber, que las ideas de por sí tengan el poder para determinar la realidad. Ésta es una postura «idealista», como diría Marx con referencia a la dialéctica hegeliana. Regresamos a este punto más adelante.

Schneider et al. (2010:511-512) identifican cinco corrientes de pensamiento que han nutrido la escuela de decrecimiento: una culturalista asociada con el trabajo de antropólogos en el Sur global quienes critican la idea de que los países del Sur deben seguir el camino del Norte; la segunda se asocia con las luchas por profundizar la democracia; la tercera con ecología; la cuarta con estilos de vida minimalistas, artísticos y pacíficos; y la quinta con la economía ecológica. Demaria et al. (2013) identifican las mismas corrientes de pensamiento y agregan lo que podemos llamar el «postestructuralismo verde», el cual consiste en un análisis crítico del discurso dominante de desarrollo.

Siguiendo a Martínez Alier et al. (2010), un esquema más sencillo para nuestros propósitos agrupa las raíces filosóficas de la escuela de decrecimiento en dos principales corrientes de pensamiento. La primera tiene que ver con la ecología y su hibridación con la economía política, dando lugar a la emergencia de dos nuevas áreas de investigación interrelacionadas: economía ecológica y ecología política. «En la economía ecológica», plantean Guha y Martínez Alier (1997), «el estudio de los temas distributivos constituye un nuevo campo de investigación, que llamamos recología política». Ya mencionamos algunos de los contribuidores más importantes de la economía ecológica, empezando con Georgescu-Roegen.

En cuanto a la ecología política, uno de los precursores más influyentes para la escuela de decrecimiento es André Gorz, un marxista de la corriente 
existencialista sartreana quien en 1968 empezó a marcar cada vez más su distancia con algunas de las tesis centrales del marxismo (por ejemplo, el papel emancipador del proletariado), al mismo tiempo que abordó cuestiones ecológicas en su crítica de las sociedades modernas y en el desarrollo de una visión utópica basada en la cooperación voluntaria y la autodeterminación de comunidades e individuos. «El capitalismo orientado al crecimiento está muerto», declaró a mediados de los años setenta, «El socialismo orientado al crecimiento (...) refleja una imagen distorsionada de nuestro pasado, no de nuestro futuro» (Gorz, 1987 [1975]:7). Un elemento central de su análisis que sería recogido por los defensores del decrecimiento tiene que ver con el avance de las fuerzas productivas y la concomitante reducción del trabajo socialmente necesario, lo que abre la posibilidad de crear tiempo libre para toda la población, a través de la distribución equitativa del trabajo colectivo.

La segunda corriente principal de pensamiento que ha nutrido el decrecimiento es la culturalista y el autor clave en ella es Iván Illich, un filósofo austriaco, sacerdote católico, multilingüista y doctor en historia medieval de la Universidad de Salzburgo. Illich trabajó con migrantes puertorriqueños en Nueva York a principios de los años cincuenta del siglo XX, posteriormente fue a trabajar en la Universidad Católica de Ponce, en Puerto Rico, y de allí migró a Cuernavaca, México, donde fundó en 1961 el Centro Intercultural de Documentación. A partir de esta formación y experiencia desarrolló una visión utópica postindustrial donde las personas y comunidades recuperan mayores niveles de autonomía frente el Estado y el mercado, al emplear «herramientas» que tienen una escala más accesible para la gente común, en lugar de las que implican centralización y administración vertical. Para Illich, el concepto de «herramienta» incluye no sólo aparatos simples como taladros, ollas o escobas, y no sólo grandes 
máquinas como coches o plantas eléctricas; sino también instituciones productivas (fábricas, por ejemplo) y sistemas de producción de bienes intangibles como la educación, la salud y los conocimientos (2009:20).

Illich identificó dos parteaguas en el desarrollo de las grandes herramientas modernas: la primera a principios del siglo XX cuando éstas empezaron a crecer y consolidarse, lo que resultó en mejoras tangibles (con múltiples contradicciones) en, por ejemplo, la tasa de mortalidad infantil, la esperanza de vida, la movilidad independiente y el alfabetismo; y la segunda, después de la Segunda Guerra Mundial cuando el tamaño y la burocratización de las instituciones conllevaron a una serie de conductas perversas y de efectos secundarios que progresivamente eclipsaron los beneficios, tales como la medicina adictiva, las cesáreas por conveniencia, la dependencia excesiva de servicios profesionales, el tráfico vehicular congestionado, la contaminación, y el diseño de currículos educativos para cumplir con las necesidades ideológicas del Estado y los requisitos técnicos de la industria. Ante esta situación, Illich (2009:13) aboga por una transición hacia una sociedad «convivencial», donde «la habilidad de una persona de expresarse en trabajo no requerirá como condición el trabajo forzado o el aprendizaje forzado o el consumo forzado de otra persona», y donde la gente puede redescubrir la «sobriedad alegre» y la «austeridad liberadora».

El trabajo de Illich ha tenido gran impacto no solamente en el pensamiento de los defensores del decrecimiento, sino también y más generalmente en la emergencia de una escuela de pensamiento estrechamente relacionada: el posdesarrollo. Según Latouche (2008:16), «el decrecimiento es simplemente un estandarte tras el cual se agrupan aquellos que han procedido a una crítica radical del desarrollo y que quieren diseñar los contornos de un proyecto alternativo para una política del postdesarrollo». 
Gudynas (2014:63) considera que «el postdesarrollo en sentido estricto sería la abreviación para indicar una crítica postestructuralista del desarrollo». De acuerdo con lo anterior, Escobar (2005:18) señala que «la noción del posdesarrollo proviene directamente de la crítica postestructuralista». En esta conexión, el postestructuralismo se refiere al acercamiento cuyo enfoque es el análisis del discurso. Como afirma Escobar (1996:326), «el postestructuralismo se enfoca en el papel del lenguaje en la construcción de la realidad social; trata al lenguaje no como un reflejo de la 〈realidad sino como constitutivo de ella».

Desde una perspectiva materialista, si bien este acercamiento puede servir para desnudar los discursos de poder, puede también conducir a la trampa del idealismo, mencionado arriba, donde se supone que, para cambiar el mundo, sólo hay que cambiar nuestra manera de pensar o cambiar nuestros valores. Una ilustración: «La apuesta por el decrecimiento (...) Consiste en pensar que la atracción por la utopía convivencial (...) puede favorecer una «descolonización del imaginario» y suscitar suficientes comportamientos 〈virtuosos〉 a favor de una solución razonable: la democracia ecológica» (Latouche, 2008:246-247). Peor aún cuando las ideas asumen explícitamente la forma de agencia: «El imaginario social se pone manos a la obra y acomete explícitamente la transformación de las instituciones existentes» (Latouche, 2008:175). Desde luego, un cambio de valores es necesario para superar los grandes problemas sociales y ecológicos, pero desde nuestro punto de vista este cambio no es tanto un punto de partida o solución en sí mismo, sino más bien algo que se construye sobre la marcha de las luchas sociales.

Es importante señalar que el posdesarrollo va más allá de la deconstrucción de los discursos de las poderosas agencias de desarrollo a nivel mundial; al rechazar el discurso y las prácticas del desarrollo desde arriba, 
llega a ciertas conclusiones sobre cómo se debe impulsar mejoras en las condiciones de vida en el ámbito local. «Las propuestas locales», afirman Esteva y Prakash (1997:285), «si son concebidas por comunidades enraizadas en lugares específicos, reflejan el pluralismo radical de las culturas y la cosmología singular que define cada cultura». Sobre esta línea, el posdesarrollo apuesta por las organizaciones y movilizaciones locales vinculadas entre sí en redes que operan en escalas mayores; apuesta por la recuperación y desarrollo de los conocimientos y tecnologías indígenas y por la defensa de los territorios y bienes comunes con significado histórico-cultural. En estos sentidos, recupera - aunque rara vez de manera explícita- una visión anarquista de un mundo poscapitalista.

Regresando al decrecimiento, ¿cómo se puede definir? Schneider et al. (2010:517) afirman que «el decrecimiento no es sólo un proyecto científico sino también parte de un movimiento social más amplio que funciona sobre la esperanza de que podamos reducir el tamaño [de la economía] de manera equitativa y democrática». En la misma vena, Martínez Alier et al. (2010:1742) argumentan que «el decrecimiento sustentable es tanto una consigna asociada con los movimientos sociales y ambientales, como un concepto emergente en círculos académicos e intelectuales». Como consigna, se aplica más a los movimientos del Norte; y éstos se ven como los principales aliados de las luchas socioambientales en el Sur por la defensa y recreación de los bienes comunes, o lo que Martínez Alier (2011) ha denominado «el ecologismo de los pobres».

Algunos de los defensores del decrecimiento sugieren que, mientras el Norte decrece, será necesario en el Sur fomentar el crecimiento de algunos bienes colectivos (que ocupan materiales y energía) para combatir la pobreza y satisfacer las necesidades básicas de toda la población, sobre todo 
en las áreas de salud y educación (véase Martínez Alier et al., 2010:1743). Otros, por ejemplo Latouche (2008:225-226), sugieren que al tomar el camino del posdesarrollo en el Sur simplemente no implica crecimiento como tal. Por otra parte, todos los defensores del decrecimiento están de acuerdo en que la clave para superar la pobreza en términos materiales es la redistribución, dentro y entre los países. Sobre esta línea, los promotores del decrecimiento exigen justicia ambiental a nivel global, donde el Norte reconoce su deuda ecológica con el Sur debido a la explotación colonial del pasado, el intercambio ecológico desigual y el acaparamiento de los bienes comunes globales no (bien) regulados, como la atmósfera en su función de sumidero para los gases de efecto invernadero.

Los defensores del decrecimiento tienden a ser ambivalentes con respecto a la cuestión de agencia. Latouche, por ejemplo, declara que «la lucha de clases se acabó» (2008:240), que el proletariado no tiene un papel emancipador y que «la transformación de la sociedad exige en la actualidad la participación de toda la población» (énfasis agregado, 2008:239). Él asigna un papel central al voluntarismo individual, señalando que existen «dos vías individuales para decrecer: la primera es consumir menos, la sobriedad; la segunda, autoproducir e intercambiar según la lógica del donativo» (2008:101).

En cuanto al Estado, también hay ambivalencia y diversas posturas. Muchos se oponen o retan a algunas instituciones, al mismo tiempo que proponen actuar dentro de las existentes. Como señala Demaria et al. (2013:203), «mientras algunos asumen una postura anarquista tradicional a favor del abandono del Estado, otros creen que se debe mantener y mejorar el Estado». Por otra parte, los defensores del decrecimiento generalmente asumen una postura anticapitalista, aunque no pongan énfasis en ésta. En efecto, Foster (2002) acusa a Latouche de «tratar de eludir la 
cuestión del capitalismo». En respuesta, Latouche (2008:168) señala que «si no insisto en la crítica específica al capitalismo, es porque me parece inútil derribar una puerta abierta». Por otra parte, opina que el reto no es sólo salir del capitalismo, sino también acabar con la sociedad productivista, con referencia al productivismo del «socialismo real».

\section{Perspectivas ecomarxistas}

Los ecomarxistas también critican el desarrollo antiecológico de la ex Unión Soviética y de China (véase por ejemplo O'Connor, 1998:255-265; Williams, 2010:170-173). Kallis (2017) considera que estas experiencias son mejor clasificadas como «capitalismo estatal» y critica a los ecomarxistas quienes todavía creen en —o que son agnósticos con respecto a — las posibilidades de realizar crecimiento económico ecológicamente sustentable bajo un sistema socialista. Desde su perspectiva, el socialismo debe ser orientado a la satisfacción de los valores de uso (determinados democráticamente) que expresan necesidades humanas, y esto no implica crecimiento en el flujo de materiales y energía, mucho menos el crecimiento del PIB (una medida de valores de cambio).

Según Foster (2011), es un error usar un concepto abstracto como el crecimiento económico como blanco de críticas, cuando el problema dimana más específicamente de la dinámica de acumulación de capital: «Aunque el concepto de decrecimiento es valioso en el sentido ecológico, sólo puede adquirir un sentido genuino como parte de una crítica de la acumulación de capital y parte de la transición a un orden sustentable, igualitario y comunal» (Foster, 2011). 
James O'Connor (1998:331) define el socialismo ecológico en los siguientes términos: «Teorías y movimientos que buscan subordinar el valor de cambio al valor de uso y el trabajo abstracto al trabajo concreto, es decir, que buscan organizar la producción alrededor de las necesidades (incluso las necesidades de desarrollo personal de los trabajadores), no de las ganancias». Löwy (2015:7) añade que esta concepción debe suponer la propiedad colectiva de los medios de producción, planeación democrática que posibilita que la sociedad defina sus prioridades de inversión y producción, y una nueva estructura tecnológica.

Por lo general, se descarta desde una perspectiva ecomarxista la posibilidad de decrecer dentro del sistema capitalista, por las razones esbozadas en la sección anterior. Se ve la necesidad no sólo de decrecer, sino también de transformar la sociedad al reorientar el modo de producción a la satisfacción de necesidades básicas, incluso con respecto a un medio ambiente sano. Otra necesidad humana básica es la de tener tiempo libre para el «florecimiento humano», como diría Julio Boltvinik. Pues, como señaló Marx (1981:958-959), «el reino de libertad realmente empieza sólo donde termina el trabajo determinado por la necesidad y los fines exteriores». Por tanto, la reducción del día de trabajo es un prerrequisito básico para eventualmente realizar el verdadero reino de libertad, concebido en términos del desarrollo de los poderes humanos como un fin en sí mismo.

Löwy (2015:51) visualiza un cambio gradual, donde cada victoria colectiva es un paso en la dirección correcta en tanto avanzan los procesos de concientización y organización. Williams (2010) implica algo parecido en su esbozo de algunos de los proyectos alrededor de los cuales se pueden organizar luchas sociales hoy día. Por ejemplo: una transición energética desde la actual dependencia de los combustibles fósiles a las fuentes renovables de energía; 
la construcción de mejores sistemas públicos de transporte para conectar centros urbanos con trenes rápidos y, dentro de las ciudades, trenes, camiones y la remodelación de la infraestructura urbana para mejor acomodar a los ciclistas, patinadores y peatones; la defensa de la salud pública frente a la contaminación del medio ambiente; y la reducción de las horas de trabajo para enfrentar los problemas de desempleo y subempleo, y para crear una sociedad que privilegie el tiempo libre sobre el consumo de bienes materiales.

Al mismo tiempo, a Williams (2010:179) no se le escapa una de las percepciones centrales del pensamiento marxista, a saber, que los estados de equilibrio se interrumpen por momentos de ruptura, no sólo en la historia humana que marca transiciones de un modo de producción a otro, sino también en la historia natural, donde se observan largos periodos de equilibrio y evolución, interrumpidos por cambios abruptos (véase York y Clark, 2011). De esta manera, Williams puede visualizar rupturas decisivas en la lucha por la transición a una forma de ecosocialismo, por ejemplo: la abolición del derecho de propiedad privada sobre la tierra y los principales medios de producción (2010:180); la redistribución de la tierra, de tal manera que los pobres urbanos del Sur se animen a regresar al campo para practicar la agroecología (2010:224); la prohibición de los anuncios y la mercadotecnia, y relacionado con esto, la eliminación de empaques/embalajes superfluos (2010:219), entre otras.

Desde esta perspectiva, las acciones individuales voluntarias no son suficientes para realizar estos cambios. «Sólo las organizaciones colectivas masivas, la presión social y la acción pueden hacer materializar estos tipos de planes», afirma Williams (2010:163), «debido a que van en contra de los intereses de corto plazo de las corporaciones y los partidos políticos». Él y otros promotores del ecosocialismo señalan como agentes principales una posible alianza entre los movimientos obreros y los ambientalistas, 
o dicho de otra manera, entre los «rojos»y los «verdes». De acuerdo con Löwy (2015:6), aunque los ecosocialistas critican la ideología de los sectores dominantes de los movimientos obreros, «saben que los trabajadores y sus organizaciones son una fuerza indispensable para cualquier transformación radical del sistema». Lo que se requiere es un movimiento «co-revolucionario» que reúne las luchas que critican el capital desde la perspectiva de la clase trabajadora, las luchas antiimperialistas, las que critican el patriarcado y el racismo, y las luchas ambientalistas que critican las consecuencias ecológicas destructivas del desarrollo capitalista (Foster, 2011).

Por último, cabe señalar que entre los ecomarxistas hay defensores de la necesidad de hacer crecer la infraestructura de producción, transportación, comunicación y también servicios públicos en las áreas de salud, educación y seguridad social. Foster (2011), por ejemplo, argumenta que «muchos países en el Sur con un nivel muy bajo de ingresos por habitante no pueden permitirse el decrecimiento sino que pueden aprovecharse de una especie de desarrollo sustentable, orientado a las necesidades reales como el acceso al agua, alimentación, salud, educación, etcétera» (énfasis en el original). Asimismo, Schwartzman (2012) argumenta que algunas cosas deben crecer para combatir a la pobreza (por ejemplo, energía solar, agroecología), mientras otras (el complejo militar, combustibles fósiles y energía nuclear) deben decrecer. Esto requiere formas de organización que tengan un alcance más allá del ámbito local, lo que para algunos implica la necesidad de conquistar y transformar el Estado, idealmente en un contexto internacional favorable para el apoyo mutuo entre Estados poscapitalistas. En todo caso, se visualiza una transformación que empieza con la construcción de movimientos sociales y redes organizacionales que empujan por cambios radicales en las leyes del Estado y su aplicación. 


\section{Los conflictos y movimientos socioambientales en México²}

Entre los problemas ambientales que han sido reconocidos y cuantificados por las agencias ambientales del país se pueden mencionar los siguientes: $45 \%$ de las tierras de cultivo sufre algún grado de degradación del suelo, 73\% del agua superficial está contaminada, 102 acuíferos están sobreexplotados y al menos 40 acuíferos están contaminados por la actividad antropogénica. Además, 2 mil 486 especies de plantas y animales están en peligro de extinción y la deforestación avanza a una tasa de 155 mil hectáreas por año, contribuyendo así a una pérdida neta de 49 millones de hectáreas desde la mitad del siglo XX, equivalente a una cuarta parte del territorio nacional. En la Ciudad de México y otros grandes centros urbanos hay serios problemas de tráfico y contaminación del aire; las emisiones de gases de efecto invernadero van a la alza, han alcanzado el equivalente de 748 millones de toneladas de dióxido de carbono en 2010; y la generación de residuos sólidos está también en aumento, ha llegado a 41 millones de toneladas en 2011, en la que una cuarta parte fue depositada en sitios no controlados. ${ }^{3}$

La extracción de materiales en México ha aumentado de manera constante durante las últimas décadas, de 349 millones de toneladas en 1970 a mil 148 millones de toneladas en 2003 (González Martínez y Schandl, 2008:319). Esto, en un marco regulatorio ambiental laxo, cuando menos en la práctica. Si bien a partir de la promulgación de la Ley General del

\footnotetext{
${ }^{2}$ Esta sección y la siguiente toman algunos fragmentos del libro Tetreault et al. (2018) escritos por el mismo autor de este texto.

${ }^{3}$ Las estimaciones para la cantidad de agua contaminada fueron tomadas de Conagua (2001); el resto de las estadísticas presentadas en este párrafo vienen de Semarnat (2013).
} 
Equilibrio Ecológico y la Protección al Ambiente (LGEEPA) en 1988, se ha construido un entramado de leyes y agencias gubernamentales ambientales en los tres niveles de gobierno cada vez más complejo e incluso con una secretaría de medio ambiente a nivel nacional, lo cierto es que las políticas ambientales en México parecen mucho más fuertes sobre el papel que en la práctica. No se trata sólo de subfinanciar las políticas ambientales, el imperativo de atraer y retener las inversiones privadas se traduce en lo que McCulligh (2017) llama «corrupción institucionalizada» para referirse no a actos individuales de corrupción, sino a una tendencia generalizada y sistemática de subordinar las leyes ambientales a los intereses de corto plazo del sector privado.

En lo que concierne a la contaminación industrial de cuerpos de agua, esta tendencia se manifiesta en un proceso de estrecha consultación con el sector privado en el diseño de las normas, un número restringido de sustancias tóxicas reguladas y la escasa aplicación de la normatividad (McCulligh, 2017). El resultado de todo esto se expone en un informe de la Comisión para la Cooperación Ambiental (CCA) sobre las emisiones de sustancias tóxicas emitidas a aguas superficiales reportadas en Canadá, Estados Unidos y México, donde se puede constatar que las empresas mexicanas han reportado mucho menos de $1 \%$ de la totalidad de las emisiones para los tres países (CCA citado en McCulligh, 2017:223).

Algo parecido pasa en el sector minero. Un estudio de la CCA (2018:9) revela que el volumen de los contaminantes reportados por el sector minero en México sólo representa 0.84\% del total en América del Norte en 2013. Más allá de lo revelado por estos datos, el hecho de que se permite la utilización de tecnologías altamente destructivas al medio ambiente, tales como las minas a cielo abierto y el beneficio por medio de la cianuración, 
evidencia el sesgo en las políticas ambientales a favor del capital extractivo. En el caso de la mina San Xavier en el estado de San Luis Potosí, propiedad de la compañía canadiense New Gold (antes Metallica Resources), es notoria la imposición del proyecto a pesar de la inconformidad de la población local y la resistencia organizada, la existencia de un área natural protegida y fallos jurídicos en su contra. Asimismo, gracias al trabajo investigativo de Garibay y sus colaboradores (2014), el caso de la mina Peñasquito en el estado de Zacatecas también ha llegado a ser notorio, entre otras razones porque dichos investigadores descubrieron que Conagua modificó su cálculo de la tasa de recarga del acuífero Cedros aumentándolo por un factor de más de cinco para poder otorgar una concesión de agua a la empresa canadiense Goldcorp en volúmenes conmensurables con la demanda de su proceso de beneficio, afectando así a la población cercana que depende del agua subterránea para sus actividades agropecuarias y para satisfacer sus necesidades básicas.

Ante atropellos de este tipo, los conflictos socioambientales se han multiplicado a lo largo del país. Fernanda Paz (2012) detectó 95 entre mayo de 2009 y mayo de 2011, 39\% relacionado al agua, 25\% a tierras agrícolas y territorios, y 15\% a áreas naturales protegidas. En un inventario más abarcador en el tiempo, Víctor Toledo y sus colaboradores (2015) detectaron 298 conflictos socioambientales entre septiembre de 2009 y marzo de 2013, vinculándolos a la minería (79), la infraestructura hidráulica (65), los proyectos energéticos (52), el desarrollo urbano (26), la silvicultura (19), la biotecnología (18), el turismo (17), los desechos peligrosos (14) y la agricultura (8). Para marzo de 2018 este inventario tenía registrados 560 casos.

Estos conflictos típicamente suceden entre grandes corporaciones que cuentan con el respaldo del Estado y las poblaciones afectadas quienes se 
organizan a nivel local y construyen alianzas con los elementos progresistas y radicales de la sociedad civil. Los movimientos de resistencia despliegan diversas formas de acción colectiva a través de redes de organización que operan en distintos ámbitos: regional, nacional e internacional. En muchos casos, son las agencias gubernamentales quienes toman el liderazgo en la promoción de grandes y controvertidos proyectos de desarrollo, particularmente en el sector petrolero y para la construcción de represas y carreteras. El capital y el Estado promueven proyectos ecológicamente destructivos bajo las banderas de «progreso», «crecimiento económico» y «modernización»; mientras que los discursos de resistencia hablan de la defensa de medios de vida en el campo, la protección de entornos ambientales sanos, los derechos humanos, los bienes comunes y la diversidad biocultural.

Se han construido redes de resistencia al despojo en varias regiones de México. Por ejemplo: en Ayala y otros municipios del estado de Morelos, donde los pobladores de 30 núcleos agrarios han organizado y construido alianzas con sujetos universitarios en contra del Proyecto Integral Morelos, que consiste en un gasoducto, un acueducto y dos plantas termoeléctricas; en la Sierra Norte de Puebla, donde múltiples organizaciones campesinas, indigenas y de la sociedad civil han unido fuerzas en oposición a un conjunto de proyectos hidroeléctricos, minería y fracking; y en Guerrero y Michoacán, donde varias comunidades indígenas han creado sus propios cuerpos policiacos para enfrentar el crimen organizado y para poner fin a la explotación ilegal de bosques y minerales.

En otros estados también se han construido movimientos de resistencia a las actividades extractivas y a los megaproyectos de desarrollo en zonas predominantemente indígenas. En el estado de Chiapas, los cinco 
caracoles que comprenden el territorio zapatista sirven como un baluarte contra la penetración de proyectos de inversión privada y como un modelo para la construcción de sociedades poscapitalistas. En Oaxaca, 418 de los 570 municipios del estado se gobiernan por usos y costumbres, lo que constituye una base institucional para sostener las luchas en contra de los proyectos de minería, represas y parques eólicos. En el estado de Sonora, los yaquis han luchado durante años en contra de la construcción y ahora el uso del Acueducto Independencia, diseñado para transferir agua de su territorio ancestral hacia la ciudad de Hermosillo. Y en el centro-norte del país, los huicholes (wixárikas) se han movilizado, junto con sus aliados de la sociedad civil, para oponerse a los planes de la compañía minera canadiense First Majestic Silver de minar plata en el territorio sagrado de Wirikuta, en el estado de San Luis Potosí.

En el ámbito nacional, destacan tres redes: la Asamblea Nacional de Afectados Ambientales (ANAA), que ha reunido 130 luchas socioambientales desde su creación en 2008; el Movimiento Mexicano de Afectados por las Presas y en Defensa de los Ríos (Mapder), nacido en octubre de 2004 en el seno de la lucha en contra de la presa La Parota en Guerrero, con el objetivo de crear un espacio para la interacción y apoyo mutuo de las luchas en contra de los megaproyectos hidráulicos y la contaminación de cuerpos de agua; y la Red Mexicana de Afectados por la Minería (Rema), creada en junio de 2008 durante la quinta asamblea general del Mapder, con el propósito de reunir a los movimientos en contra de la megaminería tóxica. A su vez, estas redes y las organizaciones constituyentes están conectadas con, y forman parte de, otras redes que operan en el ámbito internacional, tales como el Movimiento Mesoamericano contra el Modelo Extractivo Minero (M4), la Red Latinoamericana contra Represas (Redlar) e International Rivers. 
Las demandas y propuestas de estas luchas son múltiples. En torno al agua, desde su nacimiento Mapder ha exigido la cancelación de los proyectos de construir grandes presas, el desmantelamiento de las presas que «durante muchas décadas han afectado y desplazado a miles de mexicanos y mexicanas, particularmente indígenas», y la reparación de daños e indemnización para los afectados (Castro, 2004:5). Propone la búsqueda de alternativas técnicas y ahorradoras para la generación de electricidad y abastecimiento de agua, mientras denuncia la debilidad de las políticas ambientales.

Además la agrupación Agua para Todos, Agua para la Vida ha organizado una campaña para modificar las leyes y normas sobre el agua. En 2015, ante la propuesta del gobierno de Enrique Peña Nieto de una nueva Ley General de Aguas, esta agrupación elaboró la «Iniciativa Ciudadana Ley General de Aguas», a través de un proceso participativo que incluía decenas de talleres a lo largo del país con la participación de más de 420 investigadores y organizaciones. Mientras la propuesta oficial buscaba incorporar nuevos mecanismos para mercantilizar y privatizar el agua, la Iniciativa Ciudadana «establece en primer lugar que el agua es un bien de la Nación (...) y que las decisiones sobre ella tendrán que ser tomadas por los pueblos y la ciudadanía en sus respectivas escalas de territorio». ${ }^{4}$ En este tono, la Iniciativa Ciudadana pone énfasis en la creación de instancias de co-gestión del agua con base en el principio de «corresponsabilidad ciudadana-comunitaria».

Podemos observar iniciativas parecidas en el sector minero, donde los afectados por la megaminería y sus aliados retan al Estado a modificar

${ }^{4}$ Citado del sitio de internet de Agua para Todos, Agua para la Vida: http://aguaparatodos. org.mx/nuestro-proceso/ 
las leyes y prácticas existentes. Sobre esta línea, la primera versión de la «Agenda Legislativa ante el modelo y la política de la minería devastadora e inconstitucional», elaborada por Rema en 2009, exige entre otras reformas las siguientes: modificar el artículo 6 de la Ley Minera para quitar la intención de dar prioridad a las actividades mineras sobre cualquier otro tipo de actividad productiva; fortalecer las agencias ambientales y las leyes ambientales; fortalecer la participación social en materia ambiental; garantizar consultas previas, libres e informadas a las comunidades amenazadas por los grandes proyectos de minería; y crear un Fondo Especial Minero para asegurar que se realice la remediación de pasivos ambientales.

\section{Reflexiones finales y conclusiones}

Según los cálculos oficiales, más de la mitad de la población de México vive en la pobreza; y si agregamos a esta cifra el porcentaje de los mexicanos que viven en condiciones de «vulnerabilidad», la cifra sube hasta incluir cuatro de cada cinco mexicanos. Es notable que la incidencia de pobreza es mayor en el campo, donde la gran mayoría de los conflictos socioambientales suceden. Más de tres quintas partes de la población rural y tres cuartas partes de la población indígena se consideran oficialmente «pobres» (Coneval, 2015). Y estas estadísticas no capturan bien lo más fundamental para el bienestar humano: un medio ambiente sano. Por el otro extremo, hay 16 empresarios mexicanos cuyas propiedades tienen valores que se cuentan en los miles de millones de dólares. La desigualdad, medida en términos del coeficiente de Gini, ubica a México como el segundo país más desigual entre los miembros de la Organización para la 
Cooperación y el Desarrollo Económicos (OCDE, 2015), después de Chile. Bajo estas condiciones, un principio central para superar la pobreza tiene que ser la redistribución, más que el crecimiento.

No se puede refutar que el crecimiento económico era uno de los pilares centrales del modelo keynesiano de desarrollo industrial por sustitución de importaciones durante el periodo de la posguerra, junto con la construcción paulatina de políticas sociales universales, lo que resultó en mejoras tangibles en las condiciones de vida para los trabajadores en el sector formal y sus familias. No obstante, hubo fuertes contradicciones, entre ellas la marginación de grandes sectores de la población campesina e indígena, y la emergencia de múltiples problemas y conflictos socioambientales.

Una conclusión que se deriva de la discusión abordada en la primera sección de este capítulo es que ya no es posible seguir aumentando el ritmo de extracción y consumo de materiales y energía, debido precisamente a estas contradicciones que han ido exacerbándose en la época neoliberal. En una economía capitalista, el motor es la acumulación de capital ad infinitum. Esto se traduce en el imperativo de realizar de manera continua el crecimiento económico; lo que a su vez implica crecientes flujos de energía y materiales, cuyos impactos no pueden ser mitigados lo suficiente sólo por mejoras en la ecoeficiencia de los procesos productivos y de consumo. Por tanto, conforme crecen las economías capitalistas destruyen con cada vez más voracidad los ecosistemas que sostienen la vida. Una economía capitalista que no crece cae en crisis; por eso es necesario transitar hacia modos de producción y consumo poscapitalistas.

En la búsqueda de alternativas de esta naturaleza se encuentran en el campo de México diversas experiencias que ejemplifican los principios 
del posdesarrollo en el ámbito local y regional. De acuerdo con Gustavo Esteva, la insurrección zapatista y la construcción de los Caracoles en Chiapas constituye una experiencia que «corresponde claramente a la descripción de [Illich de] la sociedad convivial» (2016:21). En efecto, las comunidades zapatistas han logrado un alto nivel de autonomía en sus formas de gobernanza, caracterizada por la rotación de autoridades y el trabajo colectivo. Se destaca la autoprovisión de servicios en las áreas de educación, salud, agroecología y seguridad. Otras experiencias que ejemplifican algunos aspectos del posdesarrollo corresponden a las comunidades indígenas de Oaxaca que han recuperado y actualizado formas tradicionales de gobernanza y democracia directa. De esta manera, defienden el territorio, llevan a cabo proyectos productivos colectivos, proveen servicios sociales básicos para todos los miembros de la comunidad y organizan fiestas culturales y religiosas. En Oaxaca, el eje articulador de esta dinámica es la «comunalidad», que puede ser vista como una versión indígena de «convivialidad» que ha emergido de las luchas históricas de los pueblos indios en territorios específicos del sur de México.

A nivel nacional e internacional, el decrecimiento poscapitalista sigue siendo una utopía cuya medida de realización dependerá del desenvolvimiento de las luchas sociales y políticas en diferentes escalas. Las políticas sociales desmercantilizadoras que se pueden desprender de los debates analizados en este capítulo pueden incluir: la provisión pública por tubería de agua bebible a todos los hogares, la construcción y extensión de sistemas públicos de transporte eficientes, el abastecimiento de electricidad generada por fuentes renovables que no desplacen comunidades rurales, programas para la conservación y desarrollo in situ de semillas criollas, y el fomento de la producción agroecológica de comida sana para el 
consumo local. En un plano más abstracto y ambicioso, una transición hacia el ecosocialismo implica la desmercantilización de las tres mercancías ficticias señaladas por Polanyi (1944), a saber, el dinero, la mano de obra y la tierra, esta última como abreviatura para representar todos los recursos naturales.

En México, las demandas y propuestas de los movimientos socioambientales incluyen mayor participación ciudadana en la toma de decisiones públicas, reformas redistributivas en materia ecológica, leyes ambientales más estrictas en su contenido y aplicación, y el derecho de las comunidades rurales de no aceptar en sus territorios megaproyectos de extracción y desarrollo. Además, incluyen propuestas en torno a la agroecología, la soberanía alimentaria, el manejo forestal comunitario y la diversidad biocultural. El último eslabón del argumento desarrollado en este capítulo propone que estas demandas y propuestas — así como las lecciones que se pueden derivar de las experiencias destacadas de comunidades indígenas autónomas - constituyan claves para mejorar el bienestar en el campo mexicano sin destruir el medio ambiente.

\section{Referencias}

Castro Soto, G. (2004, 6 de octubre). «Nació Mapder. « ¿El agua es nuestra, las represas la secuestran!»» Recuperado de http://www.otrosmundoschiapas.org/analisis/ ENMAPDERI.pdf

Comisión para la Cooperación Ambiental (2018). En balance. Emisiones y transferencias de contaminación en América del Norte.

Comisión Nacional de Agua (2001). Programa Nacional Hidráulico 2001-2006. 


\section{DARCY TETREAULT}

Consejo Nacional de Evaluación de la Política de Desarrollo Social (2015). Medición de la pobreza en México y en las entidades federativas, 2014.

Daly, H. (1996). Beyond growth. Beacon Press.

Delgado Ramos, G.C. (2012). «Metabolismo social y minería». Ecología Política (43), 16-20.

Demaria, F., Schneider, F., Sekulova, F. y Martínez Alier, J. (2013). «What is degrowth? From an activist slogan to a social movement». Environmental Values (22), 191-215.

Dinda, S. (2004). «Environmental kuznets curve hypothesis: a survey». Ecological Economics (49), 431-455.

Dittrich, M. y Bringuezu, S. (2010). «The physical dimension of international trade part 1: direct global flows». Ecological Economics, 69, 1838-1847.

Ehrlich, P. (1968). The population bomb. Ballantine.

Ekins, P. (2000). Economic growth and environmental sustainability: the prospects for green growth. Routledge.

Escobar, A. (1996). «Construction nature: elements for a post structuralist political Ecology». Futures, 28(4), 325-343.

Escobar, A. (2005). «El 〈postdesarrollo〉 como concepto y práctica social». En D. Mato (coord.). Politicas de economia, ambiente y sociedad en tiempos de globalización (pp. 17-31). Facultad de Ciencias Económicas y Sociales, Universidad Central de Venezuela.

Esteva, G. (2016). «La convivialidad y los ámbitos de comunidad: claves del mundo nuevo». Crítica, 1(2), 1-25.

Esteva, G. y Prakash, M.S. (1997). «From global thinking to local thinking». En M. Rahnema y V. Bawree (coords.). The post-development reader (pp. 277289). Zed Books. 


\section{CLAVES DE LOS CONFLICTOS SOCIOAMBIENTALES MEXICANOS}

Foster, J.B. (2000). Marx's ecology. Materialism and nature. Monthly Review Press.

Foster, J.B. (2002). Ecology against capitalism. Monthly Review Press.

Foster, J.B. (2011). «Capitalism and degrowth: an impossibility theore». Monthly Review, 62(08).

Foster, J.B., Clark, B. y York, R. (2010). The ecological rift. Capitalism's War on the Earth. Monthly Review Press.

Garibay, C., Boni, A., Panico, F. y Urquijo, P. (2014). «Corporación minera, colusión gubernamental y desposesión campesina. El caso de Goldcorp Inc. en Mazapil, Zacatecas». Desacatos (44), 113-142.

Georgescu-Roegen, N. (1971). The entropy law and the economic process. Harvard University Press.

González Martínez, A.C. y Schandle, H. (2008). «The biophysical perspective of a middle income country: material flows in Mexico». Ecological Economics (68), 317-327.

Gorz, A. (1987). Ecology as politics. Pluto press.

Gudynas, E. (2014). «El posdesarrollo como crítica y el Buen Vivir como alternativa». En C.C. Delgado Ramos (coord.), Buena Vida, Buen Vivir: imaginarios alternativos para el bien común de la humanidad (pp. 61-95). Universidad Nacional Autónoma de México.

Guha, R. y Martinez Alier, J. (1997). Varieties of environmentalism. Earthscan. Illich, I. (2009). Tools for conviviality. Marion Boyars.

Kallis, G. (2017). «Socialism without growth». Capitalism Nature Socialism. DOI: 10.1080/10455752.2017.1386695

Krausmann, F., Gringrich, S., Eisenmenger, N., Erb, K.H., Haberl, H. y Fischer Kowalski, M. (2009). "Growth in global material use, GDP and population during the 20th Century». Ecological Economics (68), 2696-2705. 


\section{DARCY TETREAULT}

Latouche, S. (2008). La apuesta por el decrecimiento. ¿Cómo salir del imaginario dominante? Icaria; Antrazyt.

Löwy, M. (2015). Ecosocialism. A radical alternative to capitalist catastrophe. Haymarket.

Martínez Alier, J. (1994). De la economía ecológica al ecologismo popular. Icaria.

Martínez Alier, J. (2011). El ecologismo de los pobres. Conflictos ambientales y lenguajes de valoración. Icaria.

Martínez Alier, J. y Walter, M. (2016). «Social metabolism and conflicts over extractivism». En F. Castro, B. Hogenboom y M. Baud (eds.). Environmental governance in Latin America (pp. 58-85). Palgrave Macmillan.

Martínez Alier, J., Pascual, U., Vivien, F.D. y Zaccai, E. (2010). «Sustainable de-growth: mapping the context, criticisms and future prospects of an emergent paradigm». Ecological Economics (69), 1741-1747.

Marx, K. (1973). Grundrisse. Peguin Classics.

Marx, K. (1977). Capital. Volume I. Vintage Books.

Marx, K. (1991). Capital. Volume III. Peguin Classics.

McCulligh, C. (2017). Alcantarilla del progreso: industria y estado en la contaminación del río Santiago en Jalisco [tesis de Doctorado]. Centro de Investigaciones y Estudios Superiores en Antropología Social, Guadalajara, México.

Meadows, D., Medows, D. y Randers, J. (1972). The limits to growth: a report for the club of Rome's project on the predicament of mankind. Universe Books.

O'Connor, J. (1998). Natural causes. Essays in ecological Marxism. The Guilford Press.

Panayotou, T. (1995). «Environmental degradation at different stages». En I. Ahmed y J.A. Doeleman (eds.), Beyond Rio: the environmental crisis and sustainable livelihoods in the Third World. Macmillan Press. 


\section{CLAVES DE LOS CONFLICTOS SOCIOAMBIENTALES MEXICANOS}

Paz, M.F. (2012). «Deterioro y resistencias: conflictos socioambientales en México». En D. Tetreault, H. Ochoa y E. Hernández (eds.). Conflictos socioambientales y alternativas de la sociedad civil (pp. 27-47). ITESO.

Polanyi, K. (1944). The great transformation. The political and economic origins of our time. Beacon Press.

Sachs, W. (1993). «Global ecology and the shadow of «development»». En W. Sachs (ed.), Global ecology (pp. 3-21). Zed Books.

Schneider, F., Kallis, G. y Martinez Alier, J. (2010). «Crisis or opportunity? Economic degrowth for social equity and ecological sustainability». Journal of Cleaner Production (18), 511-518.

Schwartzman, D. (2012). "A critique of degrowth and its politics». Capitalism Nature Socialism, 23(1), 119-125.

Secretaría de Medio Ambiente y Recursos Naturales (2013). Informe de la situación del medio ambiente en México. Compendio de estadísticas ambientales. Indicadores clave y de desempeño ambiental. Edición 2012.

Stern, D. (1998). «Progress on the environmental Kuznets curve?». Environment and Development Economics (3), 173-196.

Tetreault, D., McCulligh C. y Lucio, C. (coords.) (2018). Despojo, conflictos socioambientales y alternativas en México. Miguel Ángel Porrúa/Universidad Autónoma de Zacatecas.

Toledo, V., Garrido, D. y Barrera Bassols, N. (2015). «The struggle for life socio-environmental conflicts in Mexico». Latin American Perspectives, 204(42), 133-147.

Verma, R. (2017). «Gross National Happiness: meaning, measure and degrowth in a living development alternative». Journal of Political Ecology (24), 476-490.

World Commision on Environment and Development (1987). Our common future. Oxford University Press. 


\section{DARCY TETREAULT}

Williams, C. (2010). Ecology and socialism. Solutions to capitalist ecological crisis. Haymarket.

York, R. y Clark, B. (2011). The science and humanism of Stephen Jay Gould. Monthly Review Press. 